\title{
Changes in cardiorespiratory fitness and activity levels over the first year after discharge in ambulatory persons with recent incomplete spinal cord injury
}

\author{
Matthijs F. Wouda $\mathbb{D}^{1} \cdot$ Eivind Lundgaard ${ }^{1} \cdot$ Frank Becker $\mathbb{1}^{1,2} \cdot$ Vegard Strøm $^{1}$ \\ Received: 4 February 2020 / Revised: 24 June 2020 / Accepted: 26 June 2020 / Published online: 9 July 2020 \\ (c) The Author(s) 2020. This article is published with open access
}

\begin{abstract}
Study design Secondary analysis of a clinical trial.

Objectives To investigate changes in cardiorespiratory fitness (CRF) and activity level in ambulatory persons with SCI during the first year after discharge from inpatient rehabilitation.

Setting Sunnaas Rehabilitation Hospital, Nesoddtangen, Norway.

Methods Thirty persons with incomplete SCI, all community walkers (25 males and 5 females, 18-69 years old) were recruited to a clinical trial of a 12 weeks home-based aerobic exercise program of either high or moderate intensity. During the last week of inpatient rehabilitation (baseline), participants performed a maximal exercise test on a treadmill (peak oxygen uptake; peak $\mathrm{VO}_{2}$ ) and a 6-min walking test (6MWT). Also, total daily energy expenditure (TDEE) and daily amount of steps were measured continuously during 7 days in the participants' homes. All tests were repeated after 3 and 12 months (post tests).

Results Twenty of the 30 clinical trial participants performed baseline and both posttests and are included in this secondary analysis. We found no statistically significant between-group differences in the time course over the first year of either peak $\mathrm{VO}_{2}, 6 \mathrm{MWT}$, or physical activity outcomes. Therefore, data from both exercise groups and the control group were merged for secondary analyses, revealing statistically significant increase over time in peak $\mathrm{VO}_{2}, 6 \mathrm{MWT}$, and TDEE. The increase over time in the average daily steps did not reach statistical significance.

Conclusions Ambulatory persons with SCI were able to increase their CRF levels over the first year after discharge from inpatient rehabilitation, despite a minimal increase in activity levels.
\end{abstract}

\section{Introduction}

Persons with spinal cord injury (SCI) often have low levels of daily physical activity $[1,2]$ and reduced cardiorespiratory fitness (CRF) [3-5]. Physical activity can be defined as any bodily movement produced by skeletal muscles that require energy expenditure [6]. CRF reflects

Supplementary information The online version of this article (https:// doi.org/10.1038/s41393-020-0514-7) contains supplementary material, which is available to authorized users.

Matthijs F. Wouda

Matthijs.wouda@sunnaas.no

1 Department of Research, Sunnaas Rehabilitation Hospital, Oslo, Norway

2 University of Oslo, Institute of Clinical Medicine, Oslo, Norway the integrated ability to transport oxygen from the atmosphere to the mitochondria to perform physical work. CRF quantifies an individual's functional capacity and can be measured directly, expressed as maximal oxygen consumption $\left(\mathrm{VO}_{2} \max \right)$ or peak oxygen consumption (peak $\mathrm{VO}_{2}$ ) [7].

Being physically active seems to increase CRF levels and improve health factors in persons with SCI [8]. After discharge from inpatient rehabilitation, physical activity levels, however, seem to decrease in persons with a complete SCI [9]. This might lead to physical deconditioning that can further exacerbate the impact of the injury and lead to an increased risk of cardiovascular disease [10].

In Norway, the specialized SCI rehabilitation units have a commitment to life-long follow-up for persons with SCI. After discharge from the first rehabilitation period, they are admitted for regular medical checkups (3-5 days inpatient 
care) depending on their needs. In addition, they can contact the hospital if they have specific issues that need solving [11]. In 2018, 125 persons with a newly acquired SCI were registered in Norway, of which 48 (38\%) had a preserved/ regained walking function without the need for walking aids when they were discharged from inpatient rehabilitation [12]. Similar to those who are wheelchair dependent, ambulatory persons with incomplete SCI seem to be inactive [13] and show lower levels of physical fitness [4, 14] compared to able-bodied (AB) persons. Those with an incomplete SCI have potentially more intact muscle groups below the injury level that can be utilized during physical activity. Therefore, persons with incomplete SCI, especially those with partially preserved or regained walking function, might have better premises to achieve high levels of physical fitness.

Previously [15], we reported that 30 ambulant persons with SCI increased their CRF, but not their physical activity levels during the first 3 months after discharge from inpatient rehabilitation. It is uncertain, however, if participants are able to maintain CRF levels and physical activity levels. Therefore, the purpose of the present study is to describe the changes in CRF and activity levels during the first year after inpatient rehabilitation in a group of ambulatory persons with incomplete SCI.

\section{Methods}

\section{Design}

Participants were originally recruited to take part in a 12week RCT, including a follow-up after 1 year [15]. They performed a home-based aerobic exercise intervention that consisted of either high-intensity interval training (HIIT), moderate-intensity training (MIT), or "treatment as usual" (control group). The MIT group was instructed to walk/run three times a week, for 45 min continuously at $70 \%$ of their peak heart rate (peak HR), while the HIIT group had to walk/run at an intensity of $85-95 \%$ of peak HR interspersed with $3 \times 3$ min recovery periods at an intensity of $70 \%$ of peak HR. The training mode was either walking or running (on a treadmill or outdoors) depending on the participants' CRF level and physical constraints. Due to ethical considerations, participants in the control group were not prescribed any aerobic exercise, but had no restrictions regarding physical training. All participants could if needed have contact with health care providers in the community (e.g., a physiotherapist). A detailed description of the recruitment, the interventions, and the dropout rate during the first 3 months after discharge has been provided in a previous paper [15].

\section{Participants}

In Norway, the average length of hospital stay from acute care until the end of inpatient rehabilitation was 116 days for traumatic SCI in 2018 [12]. Thirty participants (25 men and 5 women) were recruited during the last two weeks of inpatient rehabilitation by consecutive enrollment over a 4-year period (2013-2017). Inclusion criteria were persons between 18 and 17 years of age, with traumatic or nontraumatic SCI of all lesion levels [16] that had regained the ability to walk independently in the community ("community walkers"), defined by being able to walk on a treadmill for $5 \mathrm{~min}$ at 3 $\mathrm{km} / \mathrm{h}$ (without assistive walking aids). In addition, they had to be in their final phase of the subacute inpatient rehabilitation program at Sunnaas Rehabilitation Hospital. There were no restrictions for inclusion with regard to wheelchair use. Participants were excluded if they had significant concurrent medical conditions that might limit their CRF (e.g., psychiatric conditions, orthopedic diseases, or uncontrolled cardiopulmonary disease). Although the intended sample size $(n=45)$ was not accomplished, recruitment had to be ended due to time constraints. Due to lack of motivation $(n=6)$, pain $(n=3)$, and comorbidity $(n=1)$, 10 participants were lost to follow-up after 12 months. Thus, only 20 out of 30 participants performed all tests at baseline and at 3 and 12 months after discharge from inpatient rehabilitation.

\section{Procedures}

After medical approval for inclusion, (baseline) CRF tests were performed during the last week of the inpatient rehabilitation, comprising a maximal treadmill exercise test and a 6-min walking test (6MWT). The first week after discharge, participants' physical activity levels were monitored by wearing a portable activity monitor. This activity monitor, mounted on the right upper arm, was worn for seven consecutive days in a private home situation. Then, the 12-week intervention period started the second week after discharge. Three months (i.e., after the intervention period) and 1 year after discharge from inpatient rehabilitation, participants returned to the hospital for the CRF tests, repeating the same procedures as for the baseline tests. Physical activity was monitored during the 7 days after they had returned to the hospital.

\section{Outcome measures}

CRF was determined by measuring peak $\mathrm{VO}_{2}$ (prespecified primary outcome measure) using a computerized standard open-circuit technique breath-by-breath spirometer (Vmax 220, Sensormedics Corporation, Yorba Linda, CA, USA) 
during a maximal graded exercise test on a treadmill (Woodway PPS Med, Waukesha, WI, USA). In addition, respiratory exchange ratio (RER), peak HR (Polar M400), and blood lactate [La-] (BIOSEN C-line, Sport, EFK diagnostics, Barleben, Germany) were measured to evaluate whether criteria for maximal exercise testing were achieved; RER ( $>1.15$ ), peak HR $>85 \%$ of expected (for men: $>220-0.88 \times$ age, for females: $220-0.66 \times$ age) and [La-] $(>8.0 \mathrm{mmol} / \mathrm{L})$ [17]. A modified Sunnaas protocol for maximal exercise testing on a treadmill was used [4]. After establishing a self-selected walking speed, the inclination of the treadmill was increased every minute by $2 \%$, up to $20 \%$ inclination or until exhaustion. If exhaustion was not reached at the self-selected speed and $20 \%$ inclination, the speed was increased every minute by $0.5 \mathrm{~km} / \mathrm{h}$ until exhaustion. $\mathrm{VO}_{2}$ was time-averaged over $30 \mathrm{~s}$. The highest time-averaged value was considered peak $\mathrm{VO}_{2}$.

Participants performed the 6MWT (secondary outcome measure) to evaluate their walking capacity. We registered the number of meters walked, and the heart rate after the test $\left(\mathrm{HR}_{\text {after-test }}\right)$, measured with a heart rate monitor (Polar M400, Kempele, Finland).

Physical activity levels were measured with the SenseWear ${ }^{\mathrm{TM}} \mathrm{Pro}_{2}$ Armband (SWA) (Bodymedia Inc., Pittsburgh, PA, USA), a small activity monitor attached on the right upper arm. The total daily energy expenditure (TDEE) (kilojoule $(\mathrm{kJ})$ per minute) and the daily number of steps were measured. TDEE results were divided by 41,858 to transform TDEE into kilocalories (kcal) per minute [18]. SenseWear Professional 7.0 (software) was used to analyze the activity monitor data.

\section{Data analyses}

Statistical analyses were performed with the Statistical Package for the Social Sciences (release 25.0.0.1 SPSS Inc., Chicago, IL, USA). Descriptive results for all outcome measures at three different time points (baseline, 3 months post discharge and 12 months post discharge) are presented as means and standard deviations (SD).

We used linear mixed model analysis for repeated measurements, to fit the best model for the time course (i.e., modeling the response of the dependent variables peak $\mathrm{VO}_{2}, 6 \mathrm{MWT}$, TDEE, and daily steps, respectively). All 30 participants recruited in this study were used in this analysis. In this model, time was treated as a repeated fixed factor, allowing for individual random intercept and slope, and fitted with a variance components covariance structure. A time ${ }^{2}$ term was added to the models to test for a possible nonlinear response. Model selection was based on the Akaike information criterion (AIC) (i.e., a lower AIC represents a stronger model).
Table 1 Partcipants demographics and injury-specific characteristics at baseline and after 12 months.

\begin{tabular}{|c|c|c|}
\hline & $\begin{array}{l}\text { Baseline } \\
(n=30)\end{array}$ & $\begin{array}{l}12 \text { months } \\
(n=20)\end{array}$ \\
\hline \multicolumn{3}{|l|}{ Demographics } \\
\hline Age (years; mean (SD)) & $41(17)$ & $42(17)$ \\
\hline $\begin{array}{l}\text { Body weight }(\mathrm{kg} ; \\
\text { mean }(\mathrm{SD}))\end{array}$ & $81(16)$ & $82(18)$ \\
\hline BMI $\left(\mathrm{kg} / \mathrm{m}^{2} ;\right.$ mean $\left.(\mathrm{SD})\right)$ & $25.4(4.2)$ & $26.0(4.6)$ \\
\hline Smoking $(n)$ & 5 & 2 \\
\hline Male:Female (ratio) & $25: 5$ & $16: 4$ \\
\hline \multicolumn{3}{|l|}{ Injury-specific characteristics } \\
\hline $\begin{array}{l}\text { Traumatic:nontraumatic } \\
\text { (ratio) }\end{array}$ & $24: 6$ & $16: 4$ \\
\hline $\begin{array}{l}\text { Time since injury (days; } \\
\text { mean (SD)) }\end{array}$ & $69(29)$ & - \\
\hline \multicolumn{3}{|l|}{ Neurological level $^{a}(n)$} \\
\hline Cervical 1-8 & 18 & 12 \\
\hline Thoracic $1-5$ & 3 & 3 \\
\hline Thoracic $6-12$ & 3 & 1 \\
\hline Lumbar 1-5 & 5 & 4 \\
\hline Sacral 1-5 & $1^{\mathrm{b}}$ & - \\
\hline
\end{tabular}

$S D$ standard deviation, $B M I$ body mass index.

${ }^{a}$ Neurological injury level (International Standards for Neurological Classification of Spinal Cord Injury).

${ }^{\mathrm{b}}$ Apart from this participant (ASIA-A, SCI at level S2), all participants had an incomplete SCI, ASIA-D.

\section{Results}

Baseline demographics and injury-specific characteristics of all participants $(n=30)$ that were originally enrolled in the study [15] and of the remaining 20 participants at 12 months follow-up are described in Table 1. The characteristics of those lost to follow-up at 12 months $(n=10)$ did not seem to differ substantially from those that fulfilled all tests. None of the participants in this study reported that they had used a wheelchair during the first year after discharge.

Descriptive results from the physical exercise tests and the physical activity monitoring for each group at the different time points are presented in Supplementary Table 1. No statistically significant differences were found between the groups in the time course of either peak $\mathrm{VO}_{2}, 6 \mathrm{MWT}$, or physical activity outcomes (see Supplementary Table 2). Therefore, data from the three groups were combined in the following analyses.

Participants' mean peak $\mathrm{VO}_{2}$ (in both $1 / \mathrm{min}$ and $\mathrm{ml} / \mathrm{kg} / \mathrm{min}$ ) was higher, and the distance covered during the 6MWT was longer, at both 3 months and 12 months post discharge compared to baseline (Table 2). The activity monitoring data (i.e., TDEE and daily number of steps) revealed similar results (Table 2). 
Table 2 The mean results (with SD) from the maximal exercise test, 6MWT, and physical activity monitoring at baseline, 3 and 12 months.

\begin{tabular}{|c|c|c|c|}
\hline Physical capacity & $\begin{array}{l}\text { Baseline } \\
\text { Mean (SD) }\end{array}$ & $\begin{array}{l}3 \text { months } \\
\text { Mean (SD) }\end{array}$ & $\begin{array}{l}12 \text { months } \\
\text { Mean (SD) }\end{array}$ \\
\hline $\begin{array}{l}\text { Graded maximal } \\
\text { exercise test }\end{array}$ & $(n=29)$ & $(n=25)$ & $(n=19)$ \\
\hline Peak $\mathrm{VO}_{2}(1 / \mathrm{m})$ & $2.76(0.71)$ & $3.13(0.72)$ & $3.03(0.67)$ \\
\hline Peak $\mathrm{VO}_{2}(\mathrm{ml} / \mathrm{kg} / \mathrm{m})$ & $34.9(9.2)$ & $39.5(8.8)$ & $38.0(9.4)^{\mathrm{a}}$ \\
\hline Peak HR (beats/m) & $175(18)$ & $180(15)$ & $179(15)$ \\
\hline RER (ratio) & $1.21(0.11)$ & $1.21(0.07)$ & $1.18(0.06)$ \\
\hline$[\mathrm{La}-](\mathrm{mmol} / \mathrm{l})$ & $8.9(2.8)$ & $9.0(2.8)$ & $7.8(2.1)$ \\
\hline Maximal speed $(\mathrm{km} / \mathrm{h})$ & $5.0(1.0)$ & $5.6(1.4)$ & $5.8(2.1)$ \\
\hline Maximal angle $(\%)$ & $15.7(4.4)$ & $17.0(3.2)$ & $15.8(4.7)$ \\
\hline $6 \mathrm{MWT}$ & $(n=30)$ & $(n=25)$ & $(n=20)$ \\
\hline Distance (m) & $581(89)$ & $658(106)$ & $672(91)$ \\
\hline $\mathrm{HR}_{\text {after-test }}$ (beats/m) & $135(25)$ & $142(24)$ & $148(23)$ \\
\hline \multicolumn{4}{|l|}{ Physical activity } \\
\hline Activity monitoring & $(n=24)$ & $(n=22)$ & $(n=17)$ \\
\hline TDEE (kcal) & $2632(509)$ & $2739(412)$ & $2750(443)$ \\
\hline Daily steps & $5724(2786)$ & $5872(2330)$ & $6431(2891$ \\
\hline
\end{tabular}

$S D$ standard deviation, $\mathrm{VO}_{2}$ oxygen uptake, $H R$ heart rate, $R E R$ respiratory exchange ratio, $L a$ - blood lactate, $6 M W T$ 6-min walking test, TDEE total daily energy expenditure, $k c a l$ kilocalories.

aAt 12 months post discharge, the participants' mean body weight had increased by $2.6 \mathrm{~kg}$.

The mixed model analyses of peak $\mathrm{VO}_{2}(\mathrm{l} / \mathrm{min}$ and $\mathrm{ml} /$ $\mathrm{kg} / \mathrm{min}$ ) and 6MWT (meters) revealed statistically significant linear increases over time (Table 3). However, significant effects of time ${ }^{2}$ for these outcomes signifies a nonconstant rate of changes across time (Table 3). Plotting the estimated regression coefficients (i.e., the best-fitted models of the time course) of peak $\mathrm{VO}_{2}$ (liter/min) and 6MWT (meters) indicate a slight decline/leveling off after peaking at 3 months (Fig. 1a).

For the physical activity data, the time course analyses revealed a statistically significant linear increase over time for TDEE (kcal), but not for daily number of steps (Table 3). Figure $1 \mathrm{~b}$ depicts the estimated regression coefficients (see Table 3), suggesting a minor linear increase in both TDEE and daily steps.

\section{Discussion}

The present study is a follow-up to an RCT that included an exercise intervention. The main findings were that over the first year after discharge from inpatient rehabilitation, the participants significantly increased their peak $\mathrm{VO}_{2}$ and the distance walked during the 6MWT. The increase over time in the average daily steps did not reach statistical significance, despite a minor, but significant increase in the TDEE.
Table 3 Time course of peak $\mathrm{VO}_{2}, 6 \mathrm{MWT}$, and physical activity outcomes in the participants $(n=30)$.

\begin{tabular}{|c|c|c|c|}
\hline & Estimates & SE & $95 \%$ CI \\
\hline \multicolumn{4}{|l|}{$\begin{array}{l}\text { Peak } \mathrm{VO}_{2} \\
(1 / \mathrm{min})\end{array}$} \\
\hline$\left(\beta_{1}\right)$ Intercept & $2.79 * * *$ & 0.13 & $2.52-3.06$ \\
\hline$\left(\beta_{2}\right)$ Time & $0.48^{* *}$ & 0.12 & $0.23-0.73$ \\
\hline$\left(\beta_{3}\right)$ Time $^{2}$ & $-0.17 * *$ & 0.06 & -0.29 to -0.05 \\
\hline \multicolumn{4}{|l|}{$\begin{array}{l}\text { Peak } \mathrm{VO}_{2} \\
(\mathrm{ml} / \mathrm{kg} / \mathrm{m})\end{array}$} \\
\hline$\left(\beta_{1}\right)$ Intercept & $35.1 * * *$ & 1.6 & $31.7-38.4$ \\
\hline$\left(\beta_{2}\right)$ Time & $5.5^{* *}$ & 1.5 & $2.4-8.7$ \\
\hline$\left(\beta_{3}\right)$ Time $^{2}$ & $-2.1 * *$ & 0.7 & -3.7 to -0.6 \\
\hline \multicolumn{4}{|l|}{ 6MWT (m) } \\
\hline$\left(B_{1}\right)$ Intercept & $581 * * *$ & 16 & $547-614$ \\
\hline$\left(\beta_{2}\right)$ Time & $120 * * *$ & 25 & $72-169$ \\
\hline$\left(\beta_{3}\right)$ Time $^{2}$ & $-39^{* *}$ & 12 & -63 to -16 \\
\hline \multicolumn{4}{|l|}{$\begin{array}{l}\text { TDEE } \\
\text { (kcal) }\end{array}$} \\
\hline$\left(\beta_{1}\right)$ Intercept & $2657 * * *$ & 90 & $2472-2841$ \\
\hline$\left(\beta_{2}\right)$ Time & $84^{*}$ & 39 & $2-166$ \\
\hline \multicolumn{4}{|l|}{$\begin{array}{l}\text { Daily steps } \\
\text { (number) }\end{array}$} \\
\hline$\left(\beta_{1}\right)$ Intercept & $5670 * * *$ & 488 & $4674-6667$ \\
\hline$\left(\beta_{2}\right)$ Time & 323 & 302 & -298 to 943 \\
\hline
\end{tabular}

Estimated regression coefficients $(\beta)$, standard errors $(\mathrm{SE})$, and $95 \%$ confidence intervals $(\mathrm{CI})$ of the linear mixed effect models for repeated measurements fitted with variance components covariance structure of the time course over the first year after discharge from inpatient rehabilitation.

$\beta_{1}=$ estimates of baseline values; $\beta_{2}=$ estimates of the change per time unit; $\beta_{3}=$ estimates of the quadratic change per time unit. Time was in the model categorized as 0: Baseline; 1:3 months post discharge; and 2:12 months follow-up. As an example, the best-fitted model of the time course of peak $\mathrm{VO}_{2}=\beta_{1}+\beta_{2}$ Time $+\beta_{3}$ Time ${ }^{2}$.

$S E$ standard error, $C I$ confidence interval, $\mathrm{VO}_{2}$ oxygen uptake, $6 M W T$ 6-min walking test, TDEE total daily energy expenditure, kcal kilocalories.

*Statistically significant at a level of $* p<0.05$; **p $p<0.01$; ***p $<$ 0.001 .

\section{CRF and walking capacity}

To our knowledge, no other studies have described the development of CRF in ambulatory persons with SCI over the first year after discharge from inpatient rehabilitation. The time course of the peak $\mathrm{VO}_{2}$ measures, with the most profound increase after the first 3 months (Fig. 1a), was as expected. The participants had been exercising extensively during the first 3 months after discharge from the hospital, due to enrollment in an RCT of home-based aerobic exercise interventions [15]. Most of the participants that originally were enrolled in the control group reported that they also had been exercising considerably during the intervention period [15]. This might explain why participants in all groups increased their CRF during the first 3 months [15]. The levelling off in peak $\mathrm{VO}_{2}$ after 3 months indicates that the participants most likely continued regularly exercising, 
Fig. 1 Time course of cardiorespiratory fitness and physical activity parameters. Mixed model parameter estimates $( \pm$ standard errors of the mean) of fixed effects in the best-fitted models of the time course for a peak oxygen uptake $\left(\mathrm{VO}_{2}, 1 / \mathrm{min}\right)$ and 6 -min walking test (6MWT, $\mathrm{m}$ ), and $\mathbf{b}$ daily number of steps and total daily energy expenditure (TDEE, kilocalories; kcal), over the first year after discharge from inpatient rehabilitation in a group of ambulatory persons with spinal cord injury. a

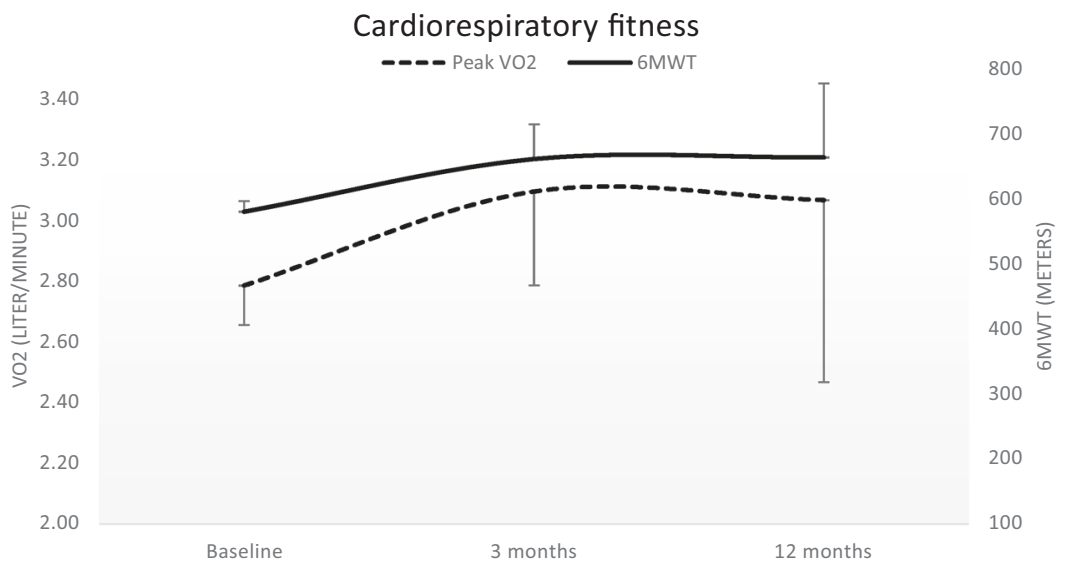

b

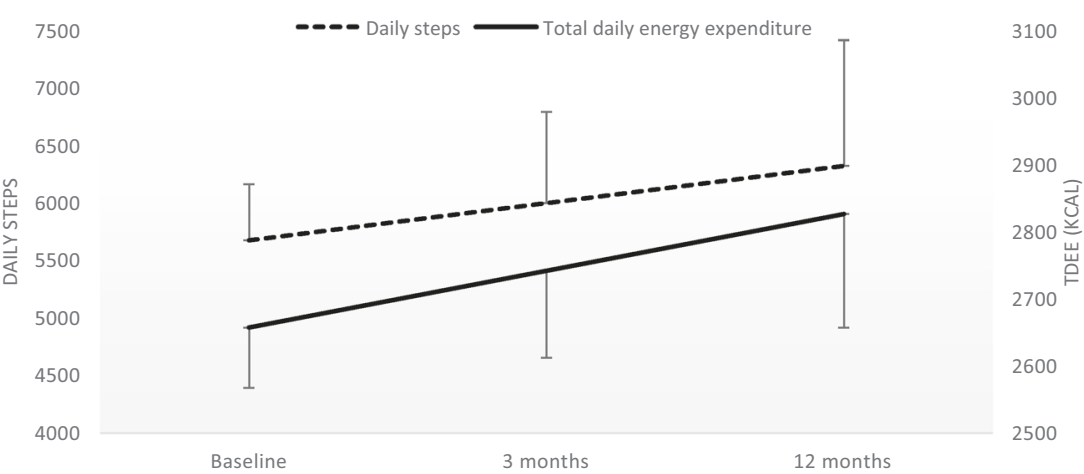

possibly at moderate or high intensity, to maintain these CRF levels also at 12 months follow-up. Unfortunately, we do not have information about which exercise was performed from 3 months until 12 months post discharge. The increased peak $\mathrm{VO}_{2}$ was concomitant to increased walking distances at the 6MWT. The observed increase of $91 \mathrm{~m}$ on the 6MWT 1 year after discharge can be considered a clinically meaningful improvement of the walking capacity of the participants [19]. However, the $\mathrm{HR}_{\text {after-test }}$ was considerably higher at 12 months follow-up, compared to baseline values (Table 2). Thus, the participants were able to walk at higher aerobic intensity one year after discharge, possibly due to the increased CRF levels. Increased lower extremity muscle strength and motor control and less spasticity might have also been contributing factors; however, we did not measure these.

The participants in the present study exhibited relatively high levels of physical fitness, as they had only $10 \%$ (4.6 $\mathrm{ml} / \mathrm{kg} / \mathrm{min})$ and $5 \%(2.6 \mathrm{ml} / \mathrm{kg} / \mathrm{m})$ lower peak $\mathrm{VO}_{2}$ levels at baseline and at 1-year follow-up, respectively, compared to (Norwegian) AB persons [20]. Furthermore, the mean distance walked during the 6MWT was, when compared with AB persons (i.e., age 20-59, males: $748 \mathrm{~m}$, females: $693 \mathrm{~m}$ ) [21], slightly reduced at baseline and at a similar level at 1-year follow-up. Most of the participants in the current study reported regularly exercising the three months prior to their SCI [15]. It is plausible that participants with high physical fitness, high interest in physical activity, and established training habits before the injury were more likely to participate in our study.

A few cross-sectional studies $[4,13,14]$ have measured fitness levels in ambulatory persons with incomplete SCI. Two studies [4, 14] on ambulatory persons with SCI, in which comparable inclusion criteria and test protocol were used, showed similar CRF levels compared to the present study.

Saraf et al. [13] included 25 persons with incomplete SCI, classified as "community walkers." Besides a significantly longer time post-injury (mean: 79 months), the injury-specific characteristics and demographics of the participants in the study of Saraf et al. are comparable to the present study. Participants in their study were, however, less physically fit and performed poorer during the 6MWT compared to the baseline results of participants in the present study. The discrepancies may partly be explained by the different inclusion criteria that were used. One of the criteria in the present study, being able to walk $5 \mathrm{~min}$ at $3 \mathrm{~km} / \mathrm{h}$ without walking aids, might demand a higher 
function of the participants, than those included in the study of Saraf et al. [13].

\section{Physical activity}

The significant time effect of TDEE could be due to the participants' gain in body weight, combined with a small increase in daily steps. However, due to the rather low accuracy of the SWA, especially when estimating energy expenditure during exercise [22], this small but significant change in TDEE cannot automatically be considered as clinically meaningful. The lack of significant time effect in the number of daily steps found in our study might be due to large between-subject variance (Table 2). As we found significantly increased CRF levels over the time course, a similar increase in daily steps could be expected. However, as demonstrated by Zisko et al. [23], in AB persons, higher CRF levels do not necessarily lead to more daily steps or an increase in TDEE. Even though the increased values did not reach statistical significance, it might be of clinical importance. The increase of about 700 steps/day from baseline to 12 months follow-up is, however, lower than a lower-bound estimate of a clinically meaningful change of $\sim 800$ steps/day in free-living walking behavior in persons with Multiple Sclerosis [24]. Barriers (e.g., lack of training facility) and facilitators (e.g., social contacts) might have influenced the participants' physical activity levels [25]. Some researchers have proposed that a reduced lower extremity muscle strength limits the physical activity level in the ambulatory SCI population [26, 27]. Participants in our study might have focused mainly on the (prescribed) aerobic exercise and thereby neglected muscle strength exercise. Ginis et al. claimed that ambulatory persons with SCI show poorer attitudes toward leisure-time physical activity than those who are wheelchair dependent [28]. As to our knowledge, only one other study has monitored physical activity levels of ambulatory persons with SCI over the first year after discharge from inpatient rehabilitation [29]. They found that participants' daily physical activity levels changed favorably during the first half-year after inpatient rehabilitation and remained stable during the second halfyear, thus not unlike our time course results.

The average level of $~ 6000$ steps per day found in our participants are somewhat below the 7100 steps/day (performed at moderate intensity) that is recommended in older adults and individuals with a disability or chronic disease [30]. They, however, emphasize that individuals living with disability and chronic illness may be more limited in their everyday activities, but could still benefit from a physically active lifestyle approximating 4600 steps/day if averaged over a week of free-living behavior [30].

\section{Limitations}

Some limitations warrant comments; first, the low sample size of 30 participants (20 at 12 months follow-up), was smaller than the intended sample size of 45 , resulting in low statistical power. The results must therefore be interpreted with caution. Second, generalizing our results should be limited to the community walking SCI population, i.e., those who are able to walk without walking aids. Third, the activity monitor (SWA) used in our study has not been validated in persons with incomplete SCI, thus caution is warranted when comparing the results on TDEE and daily steps with other populations. Fourthly, the SWA data on physical activity was collected only during seven days and thus might not reflect the participants' habitual activity levels.

To conclude, a group of ambulatory persons with SCI exhibited increased CRF levels and walking capacity over the first year after inpatient rehabilitation. This increase was most evident after the first three months, subsequent to participation in a home-based physical exercise intervention. The findings suggest that ambulatory persons with SCI may have the potential to increase and then maintain their physical fitness levels after being discharged. Physical activity levels remained rather low during the first year post discharge. This might indicate that there is a need for promoting physical activity in persons with ambulatory SCI after being discharged from inpatient rehabilitation.

\section{Data availability}

All data generated or analyzed during this study are included in this published article (and its supplementary information files).

Funding This study was (partly) sponsored by the "Birgit og Rolf Sunnaas Minnefond."

Author contributions MFW was responsible for designing the research protocol, data analysis, and writing the paper. EL was responsible for testing the participants, and he gave feedback on both the protocol and the paper. FB provided feedback on both the protocol and the paper. VS contributed to the protocol, data analysis, and the paper.

\section{Compliance with ethical standards}

Conflict of interest The authors declare that they have no conflict of interest.

Ethical approval We certify that all applicable institutional and governmental regulations concerning the ethical use of human volunteers were followed during this research. The present study was approved by the Regional Medical Ethical Research Committee. All participants signed an informed consent after being informed about the purpose, procedures, and potential risks of the study by giving written and oral information. 
Publisher's note Springer Nature remains neutral with regard to jurisdictional claims in published maps and institutional affiliations.

Open Access This article is licensed under a Creative Commons Attribution 4.0 International License, which permits use, sharing, adaptation, distribution and reproduction in any medium or format, as long as you give appropriate credit to the original author(s) and the source, provide a link to the Creative Commons license, and indicate if changes were made. The images or other third party material in this article are included in the article's Creative Commons license, unless indicated otherwise in a credit line to the material. If material is not included in the article's Creative Commons license and your intended use is not permitted by statutory regulation or exceeds the permitted use, you will need to obtain permission directly from the copyright holder. To view a copy of this license, visit http://creativecommons. org/licenses/by/4.0/.

\section{References}

1. Buchholz AC, McGillivray CF, Pencharz PB. Physical activity levels are low in free-living adults with chronic paraplegia. Obes Res. 2003;11:563-70.

2. Jorgensen S, Martin Ginis KA, Lexell J. Leisure time physical activity among older adults with long-term spinal cord injury. Spinal Cord. 2017;55:848-56.

3. Simmons OL, Kressler J, Nash MS. Reference fitness values in the untrained spinal cord injury population. Arch Phys Med Rehabil. 2014;95:2272-8.

4. Lundgaard E, Wouda MF, Strom V. A comparative study of two protocols for treadmill walking exercise testing in ambulating subjects with incomplete spinal cord injury. Spinal Cord. 2017;56:955-63

5. Janssen TW, Dallmeijer AJ, Veeger DJ, van der Woude LH. Normative values and determinants of physical capacity in individuals with spinal cord injury. J Rehabil Res Dev. 2002;39:29-39.

6. WHO. Global strategy on diet, physical activity and health; 2018. http://www.who.int/dietphysicalactivity/pa/en/.

7. Ross R, Blair SN, Arena R, Church TS, Despres JP, Franklin BA, et al. Importance of assessing cardiorespiratory fitness in clinical practice: a case for fitness as a clinical vital sign: a scientific statement from the american heart association. Circulation. 2016;134: e653-e99.

8. Nooijen CF, de Groot S, Postma K, Bergen MP, Stam HJ, Bussmann JB, et al. A more active lifestyle in persons with a recent spinal cord injury benefits physical fitness and health. Spinal Cord. 2012;50:320-3.

9. van den Berg-Emons RJ, Bussmann JB, Haisma JA, Sluis TA, van der Woude LH, Bergen MP, et al. A prospective study on physical activity levels after spinal cord injury during inpatient rehabilitation and the year after discharge. Arch Phys Med Rehabil. 2008;89: 2094-101.

10. Myers J, Lee M, Kiratli J. Cardiovascular disease in spinal cord injury: an overview of prevalence, risk, evaluation, and management. Am J Phys Med Rehabil. 2007;86:142-52.

11. Strom V, Manum G, Leiulfsrud A, Wedege P, Rekand T, Halvorsen A, et al. People with spinal cord Injury in Norway. Am J Phys Med Rehabil. 2017;96(2 Suppl 1):S99-S101.

12. Halvorsen APA, Rekand T, Olsen HC, Schaaning EE, Horn SA, Fjeldheim. Norwegian Spinal Cord Injury Registry (NorSCIR). Trondheim, Norway: St. Olavs Hospital; 2018.

13. Saraf P, Rafferty MR, Moore JL, Kahn JH, Hendron K, Leech K, et al. Daily stepping in individuals with motor incomplete spinal cord injury. Phys Ther. 2010;90:224-35.
14. Wouda MF, Wejden L, Lundgaard E, Strom V. Energetic and cardiovascular responses to treadmill walking and stationary cycling in subjects with incomplete spinal cord injury. Spinal Cord. 2016; 54:51-6.

15. Wouda MF, Lundgaard E, Becker F, Strom V. Effects of moderate- and high-intensity aerobic training program in ambulatory subjects with incomplete spinal cord injury-a randomized controlled trial. Spinal Cord. 2018;56:955-63.

16. Kirshblum SC, Burns SP, Biering-Sorensen F, Donovan W, Graves DE, Jha A, et al. International standards for neurological classification of spinal cord injury (revised 2011). J Spinal Cord Med. 2011;34:535-46.

17. Edvardsen E, Hem E, Anderssen SA. End criteria for reaching maximal oxygen uptake must be strict and adjusted to sex and age: a cross-sectional study. PLoS ONE 2014;9:e85276.

18. Mcardle WDKF, Katch VL. Exercise physiology: energy, nutrition and human performance. 6 ed. Lippincott Williams \& Wilkins; 2007.

19. Wise RA, Brown CD. Minimal clinically important differences in the six-minute walk test and the incremental shuttle walking test. COPD. 2005;2:125-9.

20. Edvardsen E, Hansen BH, Holme IM, Dyrstad SM, Anderssen SA. Reference values for cardiorespiratory response and fitness on the treadmill in a 20- to 85-year-old population. Chest. 2013;144: 241-8.

21. McKay MJ, Baldwin JN, Ferreira P, Simic M, Vanicek N, Burns J, et al. Reference values for developing responsive functional outcome measures across the lifespan. Neurology. 2017;88:1512-9.

22. Johannsen DL, Calabro MA, Stewart J, Franke W, Rood JC, Welk GJ. Accuracy of armband monitors for measuring daily energy expenditure in healthy adults. Med Sci Sports Exerc. 2010; 42:2134-40.

23. Zisko N, Stensvold D, Hordnes-Slagsvold K, Rognmo O, Nauman $\mathrm{J}$, Wisloff $\mathrm{U}$, et al. Effect of change in VO2max on daily total energy expenditure in a cohort of Norwegian men: a randomized pilot study. Open Cardiovasc Med J 2015;9:50-7.

24. Motl RW, Pilutti LA, Learmonth YC, Goldman MD, Brown T. Clinical importance of steps taken per day among persons with multiple sclerosis. PLoS ONE 2013;8:e73247.

25. Jaarsma EA, Dijkstra PU, Geertzen JH, Dekker R. Barriers to and facilitators of sports participation for people with physical disabilities: a systematic review. Scand J Med Sci Sports. 2014;24: 871-81.

26. Kim CM, Eng JJ, Whittaker MW. Level walking and ambulatory capacity in persons with incomplete spinal cord injury: relationship with muscle strength. Spinal Cord. 2004;42:156-62.

27. Shin JC, Yoo JH, Jung TH, Goo HR. Comparison of lower extremity motor score parameters for patients with motor incomplete spinal cord injury using gait parameters. Spinal Cord. 2011;49:529-33.

28. Martin Ginis KA, Papathomas A, Perrier MJ, Smith B, Group SSR. Psychosocial factors associated with physical activity in ambulatory and manual wheelchair users with spinal cord injury: a mixed-methods study. Disabil Rehabil. 2017;39:187-92.

29. Postma KB, H van Diemen T, Post M, van den Berg-Emons R. Physical activity among ambulatory people with SCI in the first year after initial inpatient rehabiliTation; a longitudinal cohort study. ISCoS. 2019.

30. Tudor-Locke C, Craig CL, Aoyagi Y, Bell RC, Croteau KA, De Bourdeaudhuij I, et al. How many steps/day are enough? For older adults and special populations. Int J Behav Nutr Phys Act. 2011;8:80. 